\title{
Minimum Depth Graph Embeddings and Quality of the Drawings: An Experimental Analysis ${ }^{\star}$
}

\author{
Maurizio Pizzonia \\ Dipartimento di Informatica e Automazione, Università Roma Tre, Italy \\ pizzonia@ia.uniroma3.it
}

\begin{abstract}
The depth of a planar embedding of a graph is a measure of the topological nesting of the biconnected components of the graph in that embedding. Motivated by the intuition that lower depth values lead to better drawings, previous works proposed efficient algorithms for finding embeddings with minimum depth. We present an experimental study that shows the impact of embedding depth minimization on important aesthetic criteria and relates the effectiveness of this approach with measures of how much the graph resembles a tree or a biconnected graph. In our study, we use a well known test suite of graphs obtained from real-world applications and a randomly generated one with favorable biconnectivity properties. In the experiments we consider orthogonal drawings computed using the topology-shape-metrics approach.
\end{abstract}

\section{Introduction}

Well known approaches for drawing graphs compute a planar embedding as an intermediate step [8] and, intuitively, the computed embedding may have a big impact on the quality of the final drawing. This motivated several research efforts to compute a planar embedding of a graph that is optimal with respect to certain cost measures (see for example [3, 11, 5]).

Recently, the concept of depth of a planar embedding has been introduced [22]. In a planar embedding, blocks (i.e. biconnected components) are inside faces, and faces are inside blocks. The containment relationships between blocks and faces induce a tree rooted at the external face. The depth of the planar embedding is the maximum length of a root-to-leaf path in this tree (see Figure 1, a rigorous definition can be found in Section 22). In [22] it is proved that it is possible to compute a planar embedding with minimum depth in linear time if the embedding of each biconnected component is given and fixed. Gutwenger and Mutzel [14] extended this result by providing an algorithm for computing embeddings with minimum depth among all planar embeddings of a graph. They also provide an algorithm that maximizes the number of vertices of the external face among all the minimum depth embeddings.

\footnotetext{
* A preliminary version of this work appeared in [21]. Work partially supported by European Commission - Fet Open project COSIN - COevolution and Self-organisation In dynamical Networks - IST-2001-33555, by European Commission - Fet Open project DELIS - Dynamically Evolving Large Scale Information Systems - Contract no 001907, by "Project ALGONEXT: Algorithms for the Next Generation Internet and Web: Methodologies, Design, and Experiments", MIUR Programmi di Ricerca Scientifica di Rilevante Interesse Nazionale, and by "The Multichannel Adaptive Information Systems (MAIS) Project", MIUR-FIRB.
} 



Fig. 1. In this example an embedded graph is shown whose blocks $A, B, C, D$ and $E$ are connected by means of the cutvertices $v, w$ and $z$. The embedding has cutfaces $f, g, h$ and $i$. The containment relationship between cutfaces and blocks is represented by a tree of depth 5 rooted at the external face $f$.

In this paper we quantify the positive effect on the final drawings of the application of embedding depth minimization techniques.

Our experiments are performed with the algorithm described in [22], which is called MinDepth throughout this paper and the results are compared with the ones obtained using Algorithm LargeDepth (detailed in Section 5) that heuristically computes planar embeddings with large depth.

For our experiments we focus on orthogonal drawings computed using the topologyshape-metrics approach [8]. This is a widely used technique for computing orthogonal drawings of general graphs. It has been extensively investigated both theoretically [15, 24, 12, 16, 17, 7, 6, 18] and experimentally [9, 2]. Its practical applicability has been demonstrated by various system prototypes [10, 19] and commercial graph drawing tools [1]. The topology-shape-metrics approach consists of three phases. The first phase takes as input a graph and computes a planar embedding possibly inserting dummy vertices to represent crossings if the graph is not planar. The second phase determines the orthogonal shape (angles) preserving the embedding. The third phase computes the coordinates of the drawing preserving both embedding and shape. With this approach the properties of the embedding computed in the first phase are crucial for the the quality of the final layout.

In our experiments, we apply the topology-shape-metrics approach where we refine the embedding computed by the planarization step using both Algorithm MinDepth and Algorithm LargeDepth. The quality of the resulting drawings is compared with respect to area, number of bends and total edge length, which are important aesthetic criteria [23]. We relate the effectiveness of embedding depth minimization with two measures, triviality and max-occupancy, which express how much a graph resembles a tree or a biconnected graph, respectively. Results show that the effectiveness of embedding depth minimization is strongly affected by the values of such measures.

Our experiments are performed on two test suites. The first is a well known set of graphs obtained from real-world applications that allows us to test the effectiveness of embedding depth minimization on realistic instances. The second is randomly generated so that graphs present specific biconnectivity properties which allow the embedding depth to vary over a wide range. This permits us to understand how effective the technique can be in a very favorable setting. 
This paper is organized as follows. Section 2 provides basic definitions. In Section 3 we define new biconnectivity-related measures. In Section 4 we analyze the test suites used in this paper for biconnectivity properties. In Section 5 we describe a heuristic algorithm for computing embeddings with large depth. In Section 6 we show the results of our experimental analysis. In Section 7 we draw the conclusions of our work.

\section{Basic Definitions}

In this section, we review basic concepts about graphs and embeddings, and give definitions that will be used throughout the paper.

Let $G$ be a connected planar graph. For simplicity, we assume that $G$ has no parallel edges or self-loops. A cutvertex of $G$ is a vertex whose deletion disconnects $G$. Graph $G$ is said to be biconnected if it has no cutvertices. A block $B$ of $G$ is a maximal subgraph of $G$ such that $B$ is biconnected. A trivial block is composed by one edge between two cutvertices with no other path between them. The block-cutvertex tree $T$ of $G$ is a tree whose nodes are in one-to-one correspondence with the blocks and the cutvertices of $G$, and whose edges connect each cutvertex-node to the block-nodes of the blocks containing the cutvertex.

An embedding $\Gamma$ of $G$ is an equivalence class of planar drawings of $G$ with the same circular order of edges around each vertex. Two planar drawings with the same embedding also induce the same circuits of edges bounding corresponding regions in the two drawings. These circuits are called the faces of the embedding.

The dual embedding $\Gamma^{\prime}$ of $\Gamma$ is the embedded graph induced by the adjacency relations among the faces of $\Gamma$ through its edges. A cutface $f$ of $\Gamma$ is a face associated with a cutvertex of $\Gamma^{\prime}$. The block-cutface tree $T^{*}$ of $\Gamma$ is the block-cutvertex tree of $\Gamma^{\prime}$. Since the dual of any biconnected embedding is biconnected, $T$ and $T^{*}$ contain the same set of block-nodes.

A planar embedding is an embedding where a face is chosen as external face. We consider the block-cutface tree of a planar embedding either rooted at the external face, if this is a cutface, or rooted at the block that contains the external face.

For a rooted tree $T$ the depth of $T$ (depth $T$ ) is the length of the longest path from the root of $T$ to one of its leaves. The diameter of a tree is the length of the longest path between any two leaves.

Let $G$ be a connected planar graph, and assume that we have a prescribed embedding for each block $B$ of $G$. We say that $\Gamma$ is block-preserving for a cutvertex $v$ if $\Gamma$ preserves the embedding of each block $B$ containing $v$, that is, the circular order of the edges of $B$ incident on $v$ is equal to their circular order in the prescribed embedding of $B$. We say that $\Gamma$ is block-preserving if it is block-preserving for all cutvertices.

Given a cutvertex $v$ of $G$ and block $B$ containing $v$, we call the pair $(B, v)$ a cutpair. The faces of block $B$ containing $v$ are called the candidate cutfaces for the cutpair $(B, v)$ since one or more of them can be cutfaces in block preserving embeddings of $G$.

\section{Biconnectivity-Related Graph Measures}

The effectiveness of depth minimization depends on the biconnectivity properties of the graph. It is easy to find families of graphs whose embedding depth cannot be changed 
by changing the embedding of their cutvertices. Trees and biconnected graphs are examples of such families. Also, graphs that are "almost trees" or "almost biconnected" permit a very small variability of the embedding depth and hence we expect the effectiveness of depth minimization techniques to be small for such instances.

We introduce the following measures for a connected graph. We call triviality of a graph the ratio between the number of the trivial blocks and the total number of edges of the graph. For each block of the graph, its occupancy is the the ratio between the number of its edges and the total number of edges of the graph. We call max-occupancy of a graph the maximum among the occupancies of its blocks.

Intuitively, the triviality is a measure of how much a graph resembles a tree. The triviality of a tree is 1 while the triviality of a biconnected graph is 0 . The max-occupancy is a measure of how much a graph resembles a biconnected graph. A tree has maxoccupancy near to 0 while a biconnected graph has max-occupancy equal to 1 .

We expect depth minimization techniques to be particularly useful on graphs that show both small triviality and small max-occupancy. Small triviality implies that most of the blocks have more than one face, i.e., the blocks contain cycles that may host part of the graph, potentially increasing the embedding depth. On the other hand, small max-occupancy implies that the graph has many blocks of small size making it possible to have many nestings and hence high depth value. Note that, the average of the occupancies of the blocks is not useful for our purposes. Consider a graph that contains one big block and many blocks of only one edge, we have high max-occupancy and low average occupancy. In such a graph, the variability of the embedding introduced by the presence of the trivial blocks have a small effect on the quality of the drawings, since aesthetic measures are largely due to the drawing of the big block.

\section{Test Suites Analysis}

The experiments described in Section 6 are performed over a test suite of about 3,000 graphs containing primarily graphs that represent real-world data and a test suite of 410 randomly generated graphs with specific characteristics.

Graphs from real-world applications. The graphs that represent real-world data are a subset of the graphs available from the GDToolkit web site [10] (ALF_CU data set) and first used in [9]. We selected the 3724 graphs with no more than 50 nodes from this set. Most of these graphs were non planar. Most of the graphs with more than 50 nodes have one big block and many very small blocks and are not well suited for testing the effectiveness of the depth minimization technique we consider.

In Figure 2(a) the distribution of the diameter of the block-cutvertex tree in the test suite is shown (average 9.21). In Figure 2(b) the distribution of the degree of the cutvertices in the block-cutvertex tree is shown. Most of the cutvertices have degree equal to 2 which is a rather low value. Figures 2(c) and 2(d) show the distribution of the maxoccupancy and of triviality respectively. Most of the graphs have large max-occupancy which implies that most have one large block encompassing a large portion of the graph. The above observations show that most of the graphs of this test suite are instances that can be considered hard for depth minimization techniques. 


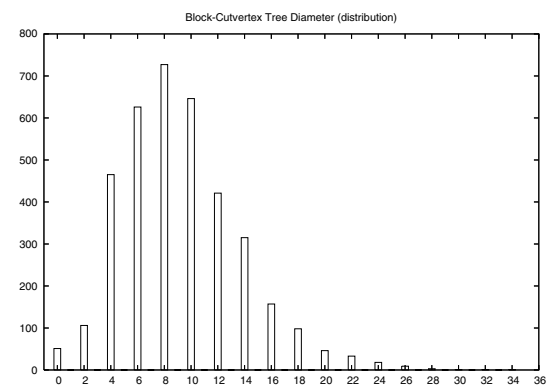

(a) Block-cutvertex tree diameter (distribution)

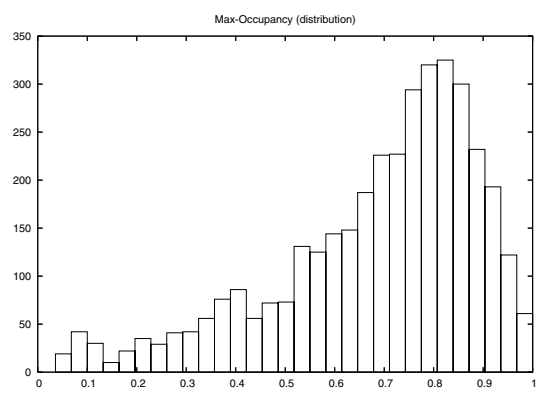

(c) Max-occupancy (distribution)

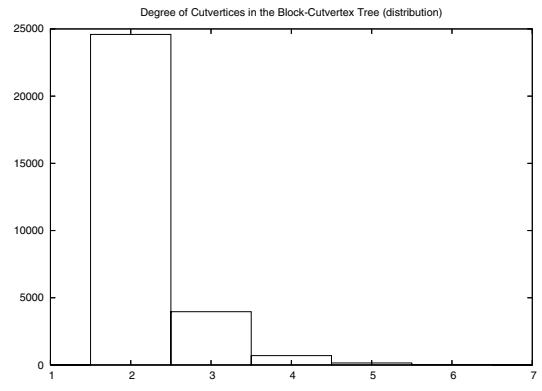

(b) Degree of cutvertices in the blockcutvertex tree (distribution)

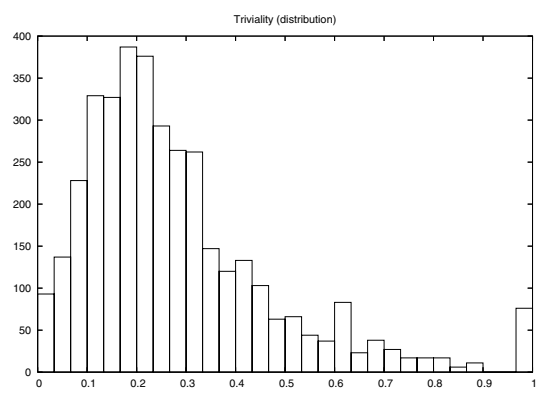

(d) Triviality (distribution)

Fig. 2. Distributions of biconnectivity-related measurements on the graphs obtained from realworld applications. Statistics are performed on graphs with up to 50 vertices.

Randomly generated graphs. We randomly generated a set of graphs that feature small triviality and small max-occupancy. The purpose of such graphs is to understand the effectiveness of embedding depth minimization in a favorable situation. We generated 410 planar graphs with number of nodes $n$ ranging between 10 and 50. For each value of $n$ we generated 10 graphs. The generation process creates graphs with the number of cutvertices between $n / 10$ and $n / 5$, for each cutvertex the number of blocks incident to it is between 2 and 5 , for each block the number of cutvertices incident to it is no more than 5. The details of the generation algorithm are given in [21].

Some statistics about the randomly generated test suite are shown in Figure 3

\section{Computing Embeddings with Minimum Depth and Large Depth}

In this section, we describe in detail Algorithm LargeDepth for computing planar embeddings with large depth preserving the embeddings of the blocks, then, to make this paper more self-contained, we briefly sketch Algorithm MinDepth whose details are described in [22].

Algorithm LargeDepth takes as input a connected planar graph $G$ with a prescribed embedding for each of its blocks, and a block $B$ of $G$. The output is an embedding $\Gamma$ 




(a) Block-cutvertex tree diameter (distribution)

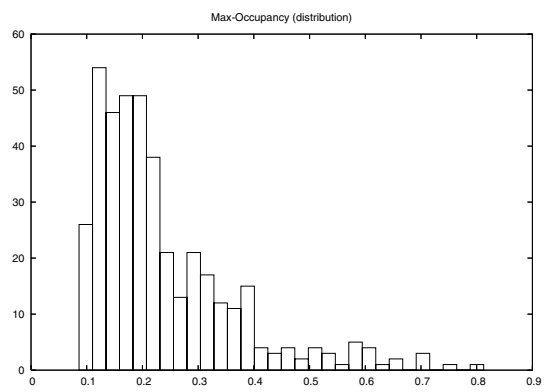

(c) Max-occupancy (distribution)



(b) Degree of cutvertices in the blockcutvertex tree (distribution)

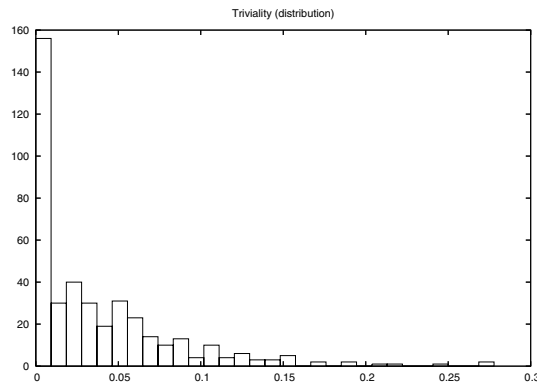

(d) Triviality (distribution)

Fig. 3. Distributions of biconnectivity-related measurements on the graphs of the randomly generated test suite

of $G$ with large depth which has the external face in $B$. The algorithm considers the block-cutvertex tree $T$ of $G$ rooted at $B$ and builds the planar embedding $\Gamma$ by means of a post-order traversal of $T$.

Given a node $x$ of $T$ (it may be a cutvertex or a block) we denote with $G(x)$ the subgraph of $G$ associated with the subtree of $T$ rooted at $x$ and with $\Gamma(x)$ the planar embedding of $G(x)$ computed by method embed $(x)$ of the algorithm.

Method embed $(x)$ takes as input graph $G(x)$ and returns a planar embedding $\Gamma(x)$ of $G(x)$ with large depth. Let $y_{1}, y_{2}, \ldots, y_{m}$ be the children of $x$ in $T$. The embedding $\Gamma(x)$ is computed by assembling the previously computed embeddings $\Gamma\left(y_{i}\right)$ of the children of $x$. Since we aim at obtaining embeddings whose block-cutface tree shows a high depth, when $x$ is a cutvertex, blocks $y_{1}, y_{2}, \ldots, y_{m}$ are embedded so that the block with the deepest embedding is inside all other blocks. The detailed description of Algorithm LargeDepth follows.

\section{Algorithm LargeDepth}

input. A connected planar graph $G$ with block-cutvertex tree $T$, a prescribed embedding for the blocks of $G$, and a block $B$. 
output. A block-preserving planar embedding $\Gamma$ of $G$ of large depth that has the external face in $B$.

The algorithm consider $T$ rooted at $B$. It computes and returns $\Gamma=\operatorname{embed}(B)$.

\section{Method embed $(B)$}

input A block $B$ of $T$.

output A block-preserving planar embedding $\Gamma(B)$ of large depth that has the external face in $B$.

\section{for all children $v$ of $B$ in $T$ do}

Let $\Gamma(v)=\operatorname{embed}(v)$.

\section{end for}

Let $\Gamma(B)$ be equal to the prescribed embedding of $B$.

for all children $v$ of $B$ in $T$ do

Modify $\Gamma(B)$ by attaching $\Gamma(v)$ into one of the candidate cutfaces for the cutpair $(B, v)$

\section{end for}

if $B$ is the root of the block-cutface tree then

choose the external face of $\Gamma(B)$ such that it is not a cutface, if possible. else

choose an arbitrary external face for $\Gamma(B)$ (since embed $(v)$ will change it)

\section{end if}

\section{$\operatorname{Method~embed~}(v)$}

input A cutvertex $v$ of $T$.

output A block-preserving planar embedding $\Gamma(v)$ of large depth that has $v$ on the external face.

\section{for all children $B$ of $v$ in $T$ do}

Let $\Gamma(B)=\operatorname{embed}(B)$.

\section{end for}

Partition the blocks that are children of $v$ in $T$ into two sets $\mathcal{B}_{T}$ and $\mathcal{B}_{N T}$ :

- $\mathcal{B}_{T}$ contains all the trivial blocks (blocks that have only one edge)

- $\mathcal{B}_{N T}$ contains all the non-trivial blocks

\section{for all blocks $B$ in $\mathcal{B}_{N T}$ do}

For each $B \in \mathcal{B}_{N T}$ select, among the candidate cutfaces for the cutpair $(B, v)$ in $\Gamma(B)$, two distinct candidate cutfaces: $f_{\text {ext }}(B), f_{\text {int }}(B)$, where possibly $f_{\text {ext }}(B)$ does not contain any block.

\section{end for}

Let $\bar{B}$ be a block in $\mathcal{B}_{N T}$ that shows the maximum of depth $\Gamma(B)$.

Let $\Gamma^{\prime}$ be $\Gamma(\bar{B})$ where all the blocks in $\mathcal{B}_{T}$ are attached into $f_{\text {int }}(\bar{B})$ and the external face of $\Gamma^{\prime}$ be $f_{\text {ext }}(\bar{B})$.

for all blocks $B$ in $\mathcal{B}_{N T}-\{\bar{B}\}$ do 
Modify $\Gamma^{\prime}$ such that it is equal to $\Gamma(B)$ where the old $\Gamma^{\prime}$ is placed into $f_{\text {int }}(B)$ and its external face is $f_{\text {ext }}(B)$.

\section{end for}

Let $\Gamma(v)=\Gamma^{\prime}$.

Algorithm LargeDepth computes deep embeddings since method embed $(v)$ computes an embedding whose depth is the maximum depth shown by the children of $v$ plus 2 times the number of the other non-trivial children of $v$.

Now we briefly sketch Algorithm MinDepth whose details are given in [22]. The algorithm starts from an arbitrary cutvertex $v$ of the block-cutvertex tree and builds an embedding that has $v$ on the external face, by means of a post-order traversal. In choosing how to embed a block and the subgraphs associated with its children, it puts the deepest sub-embeddings into the external face if possible. The result is an embedding with minimum depth among those that have $v$ on the external face. All the subtrees of the rooted block-cutvertex tree turn out to be embedded such that the corresponding block-cutface tree has minimum depth. Starting from this embedding, it is possible to compute a block preserving embedding whose block-cutface tree has minimum diameter by applying a small number of changes. The cutface with minimum eccentricity in the block-cutface tree is chosen to be external which gives a block preserving minimum depth embedding.

\section{Experimental Results}

In this section we report the results of the experiments we performed on the test suites described in Section 4 Our goal is to show how much the depth of the embeddings affects the area of the drawings, their total edge length, and their number of bends.

The graphs derived from real-world applications may be in general non-planar. According to the topology-shape-metrics approach we planarize them. The planarization heuristic 1 adopted is a well-known one described in [8] and implemented in GDToolkit. This technique does not introduce new cutvertices and hence it does not change the block-cutvertex tree of the graph.

For each graph, after the planarization step, we generated two embeddings: one with minimum depth, by means of Algorithm MinDepth, and one with large depth, by means of Algorithm LargeDepth. Algorithm LargeDepth was run, for each graph, with all possible blocks as root and the deepest among the computed embeddings wasselected.

We draw such two embeddings according to the orthogonal drawing standard introduced in [4] (simple podevsnef). The algorithm used to compute the shape minimizes the number of bends within that standard. The compaction technique used is the heuristid 2 presented in [2] which iteratively compacts along the horizontal and the vertical direction until the drawing does not change. It provides good performance in terms of area and total edge length of the drawing.

\footnotetext{
${ }^{1}$ The problem of planarizing a graph introducing the minimum number of crossings is NPhard [13].

${ }^{2}$ The problems of compacting an orthogonal drawing in order to obtain minimum area or minimum total edge length are NP-hard [20].
} 


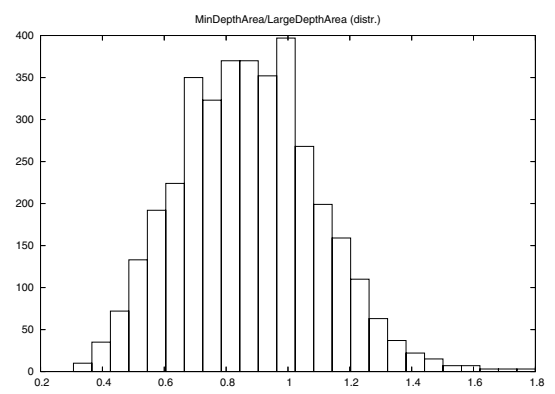

(a) $\operatorname{Area}\left(\Gamma_{\min }\right) / \operatorname{Area}\left(\Gamma_{\text {large }}\right)$



(c) $\operatorname{Bends}\left(\Gamma_{\text {large }}\right)-\operatorname{Bends}\left(\Gamma_{\min }\right)$

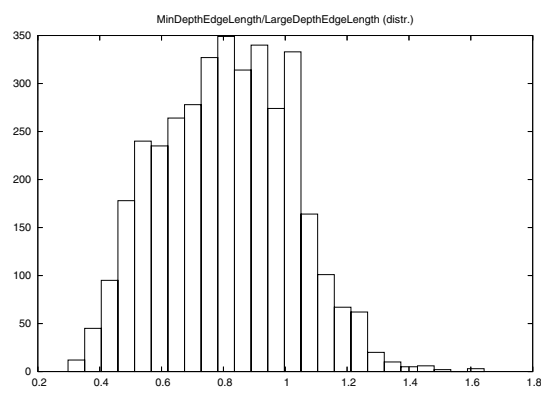

(b) $\operatorname{EdgeLen}\left(\Gamma_{\min }\right) / \operatorname{EdgeLen}\left(\Gamma_{\text {large }}\right)$



(d)

Fig. 4. Measurements performed on the test suite derived from real-world applications. The charts show the distributions of several comparative parameters.

We call $\Gamma_{\min }$ the drawing obtained from the embedding with minimum depth and $\Gamma_{\text {large }}$ the drawing obtained from the embedding with large depth. Figure 4(a) shows the distribution of the ratio between the area of $\Gamma_{\min }$ and the area of $\Gamma_{\text {large }}$. Figure 4(b) shows the distribution of the ratio between the total edge length of $\Gamma_{\min }$ and the total edge length of $\Gamma_{\text {large }}$. Figure 4(c) shows the distribution of the difference between the number of bends of $\Gamma_{\text {large }}$ and $\Gamma_{\min }$. Figure 4(d) shows $\Gamma_{\text {large }}$ and $\Gamma_{\min }$ for a graph of 34 vertices (ug31.34).

From Figure 4 we can see that even with the first test suite (the hard one) there is a clear advantage in minimizing the embedding depth. In particular, for $2 / 3$ of the graphs $\Gamma_{\text {min }}$ has better area than $\Gamma_{\text {large }}$ and there are peaks in which the area is decreased to $1 / 2$ of the area of $\Gamma_{\text {large }}$. A similar behavior may be observed for the total edge length. The average of the ratio between the area of $\Gamma_{\min }$ and $\Gamma_{\text {large }}$ is 0.87 . The average of the ratio between the total edge length of $\Gamma_{\min }$ and $\Gamma_{\text {large }}$ is 0.81 . For the number of bends the result is not so good. For most of the graphs the number of bends is unchanged. However, in some cases the number decreases up to 9 units.

We repeated the same experiments on the test suite of 410 randomly generated graphs and the corresponding charts are shown in Figures 5(a) 5(b) and 5(c) Figure 5(d) shows $\Gamma_{\text {large }}$ and $\Gamma_{\min }$ for a graph of 26 vertices. 




(a) $\operatorname{Area}\left(\Gamma_{\min }\right) / \operatorname{Area}\left(\Gamma_{\text {large }}\right)$

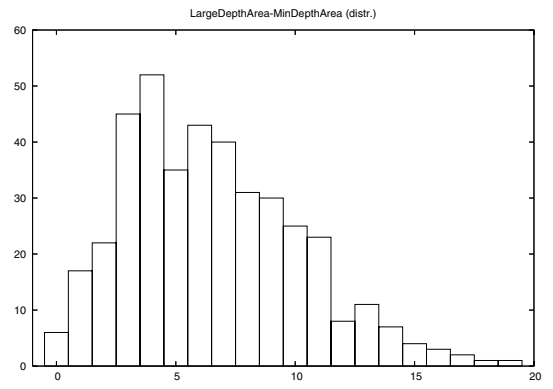

(c) $\operatorname{Bends}\left(\Gamma_{\text {large }}\right)-\operatorname{Bends}\left(\Gamma_{\text {min }}\right)$

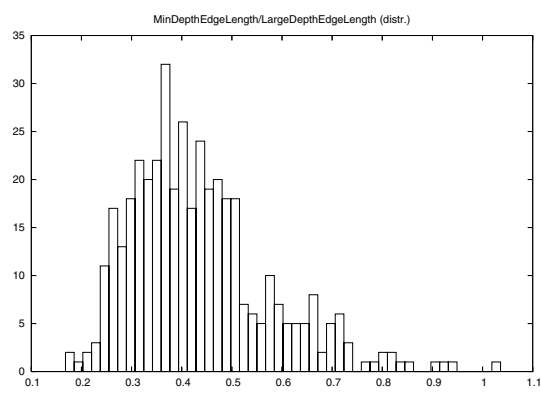

(b) $\operatorname{EdgeLen}\left(\Gamma_{\min }\right) / \operatorname{EdgeLen}\left(\Gamma_{\text {large }}\right)$
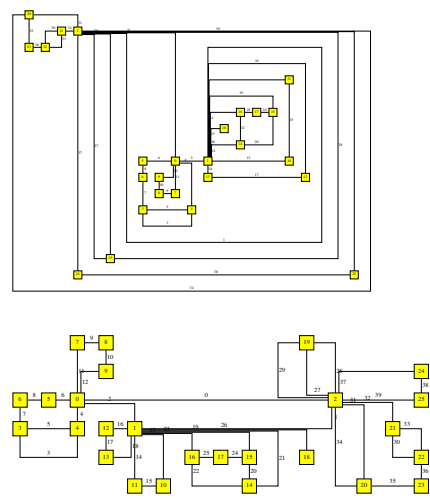

(d)

Fig. 5. Measurements performed on the randomly generated test suite. The charts show the distributions of several comparative parameters.

The results in this case are unquestionable. Almost all the considered graphs were drawn with smaller area, smaller total edge length and fewer number of bends. There are $\Gamma_{\min }$ drawings whose area is $1 / 5$ of the area of the corresponding $\Gamma_{\text {large }}$. The same holds for total edge length. The number of bends shows an average reduction of 6.5 and a peak reduction of 19. The average of the ratio between the area of $\Gamma_{\min }$ and $\Gamma_{\text {large }}$ is 0.51 . The average of the ratio between the total edge length of $\Gamma_{\min }$ and $\Gamma_{\text {large }}$ is 0.43 .

\section{Conclusions}

Our experiments prove that the embedding depth minimization technique has a positive effect on the quality of the drawings when adopted as a refinement step after the planarization phase of the topology-shape-metric approach. The effectiveness of the technique was explored using graphs obtained from real-world data, which gives results useful for application, and with graphs randomly generated that show specific biconnectivity properties, which is useful for theoretical investigation. We introduced 
two new measures to easily characterize the latter family of graphs. Also, we provided a heuristic to compute embeddings with large depth, a problem that may be subject of a more rigorous theoretical investigation.

Other interesting experimental and/or theoretical analysis can be performed in this area. For example, investigate how much better the techniques introduced in [14] perform compared with those used in this paper? Also, suppose that we randomly choose embeddings, how likely is it to obtain embeddings with small (or large) depth?

\section{References}

1. D. Alberts, C. Gutwenger, P. Mutzel, and S. Näher. AGD-Library: A library of algorithms for graph drawing. In Proc. Workshop on Algorithm Engineering, pages 112-123, 1997.

2. G. Di Battista, W. Didimo, M. Patrignani, and M. Pizzonia. Orthogonal and quasi-upward drawings with vertices of prescribed size. In Graph Drawing (Proc. GD '99), volume 1731 of Lecture Notes Comput. Sci. Springer-Verlag, 1999.

3. P. Bertolazzi, G. Di Battista, and W. Didimo. Computing orthogonal drawings with the minimum number of bends. In Workshop Algorithms Data Struct. (WADS'97), volume 1272 of Lecture Notes Comput. Sci., pages 331-344. Springer-Verlag, 1997.

4. P. Bertolazzi, G. Di Battista, and W. Didimo. Computing orthogonal drawings with the minimum number of bends. IEEETC: IEEE Transactions on Computers, 49, 2000.

5. D. Bienstock and C. L. Monma. On the complexity of embedding planar graphs to minimize certain distance measures. Algorithmica, 5(1):93-109, 1990.

6. U. Brandes and D. Wagner. Dynamic grid embedding with few bends and changes. Lecture Notes in Computer Science, 1533, 1998.

7. Ulrik Brandes and Dorothea Wagner. A Bayesian paradigm for dynamic graph layout. In Graph Drawing (Proc. GD '97), volume 1353 of Lecture Notes Comput. Sci., pages 236-247. Springer-Verlag, 1998.

8. G. Di Battista, P. Eades, R. Tamassia, and I. G. Tollis. Graph Drawing. Prentice Hall, Upper Saddle River, NJ, 1999.

9. G. Di Battista, A. Garg, G. Liotta, R. Tamassia, E. Tassinari, and F. Vargiu. An experimental comparison of four graph drawing algorithms. Comput. Geom. Theory Appl., 7:303-325, 1997.

10. Giuseppe Di Battista et al. Graph Drawing Toolkit. University of Rome III, Italy. http://www.dia.uniroma3.it/ $/ \mathrm{gdt} /$.

11. W. Didimo and G. Liotta. Computing orthogonal drawings in a variable embedding setting. In Algorithms and Computation (Proc. ISAAC '98), volume 1533 of Lecture Notes Comput. Sci., pages 79-88. Springer-Verlag, 1998.

12. Ulrich Fößmeier and Michael Kaufmann. Drawing high degree graphs with low bend numbers. In Graph Drawing (Proc. GD '95), volume 1027 of Lecture Notes Comput. Sci., pages 254-266. Springer-Verlag, 1996.

13. M. R. Garey and D. S. Johnson. Crossing number is NP-complete. SIAM J. Algebraic Discrete Methods, 4(3):312-316, 1983.

14. C. Gutwenger and P. Mutzel. Graph embedding with minimum depth and maximum external face. In Graph Drawing (Proc. GD '03), volume 2912 of Lecture Notes Comput. Sci., pages 259-272. Springer-Verlag Heidelberg, 2004.

15. J. Hopcroft and R. E. Tarjan. Efficient planarity testing. J. ACM, 21(4):549-568, 1974.

16. M. Jünger and P. Mutzel. Maximum planar subgraphs and nice embeddings: Practical layout tools. Algorithmica, 16(1):33-59, 1996. 
17. Michael Jünger, Sebastian Leipert, and Petra Mutzel. Pitfalls of using PQ-Trees in automatic graph drawing. In Graph Drawing (Proc. GD '97), volume 1353 of Lecture Notes Comput. Sci., pages 193-204. Springer-Verlag, 1997.

18. G. W. Klau and P. Mutzel. Optimal compaction of orthogonal grid drawings. In IPCO: 7th Integer Programming and Combinatorial Optimization Conference, volume 1610 of Lecture Notes Comput. Sci. Springer-Verlag, 1999.

19. Harald Lauer, Matthias Ettrich, and Klaus Soukup. GraVis - system demonstration. In Graph Drawing (Proc. GD '97), volume 1353 of Lecture Notes Comput. Sci., pages 344349. Springer-Verlag, 1997.

20. Maurizio Patrignani. On the complexity of orthogonal compaction. Computational Geometry: Theory and Applications, 19(1):47-67, 2001.

21. M. Pizzonia. Engineering of Graph Drawing Algorithms for Applications. PhD thesis, Dipartimento di Informatica e Sistemistica, University degli Studi "La Sapienza" di Roma, 2001.

22. M. Pizzonia and R. Tamassia. Minimum depth graph embedding. In M. Paterson, editor, Algorithms - ESA 2000, volume 1879 of Lecture Notes Comput. Sci. Springer-Verlag, 2000.

23. H. C. Purchase, R. F. Cohen, and M. James. Validating graph drawing aesthetics. In F. J. Brandenburg, editor, Graph Drawing (Proc. GD '95), volume 1027 of Lecture Notes Comput. Sci., pages 435-446. Springer-Verlag, 1996.

24. R. Tamassia. On embedding a graph in the grid with the minimum number of bends. SIAM J. Comput., 16(3):421-444, 1987. 